\title{
Myosins generate contractile force and maintain organization in the cytokinetic contractile ring
}

${ }^{1}$ Department of Chemical Engineering, Columbia University, New York, NY 10027, USA

${ }^{2}$ Department of Physics, Columbia University, New York, NY 10027, USA

${ }^{3}$ Department of Cellular and Molecular Physiology, Yale University, New Haven, CT 06520, USA

${ }^{4}$ Nanobiology Institute, Yale University, West Haven, CT 06516, USA

${ }^{5}$ Department of Molecular, Cellular, and Developmental Biology, Yale University, New Haven, CT 06520, USA

${ }^{6}$ Department of Molecular Biophysics and Biochemistry, Yale University, New Haven, CT 06520, USA

${ }^{7}$ Department of Cell Biology, Yale University, New Haven, CT 06520, USA

†These authors contributed equally to this work.

"To whom correspondence may be addressed. Email: bo8@ columbia.edu.

Keywords: cytokinesis, myosin II, actin, contractile ring tension, fission yeast, actomyosin contractility 


\begin{abstract}
During cytokinesis cells assemble an actomyosin contractile ring whose tension constricts and divides cells, but the ring tension was rarely measured. Actomyosin force generation is well understood for the regular sarcomeric architecture of striated muscle, but recent super-resolution studies of fission yeast contractile rings revealed organizational building blocks that are not sarcomeres but irregularly positioned plasma membrane-anchored protein complexes called nodes. Here we measured contractile ring tensions in fission yeast protoplast cells. The myosin II isoforms Myo2 and Myp2 generated the tension, with a 2-fold greater contribution from Myo2. Simulations of a molecularly detailed ring model revealed a sliding node mechanism for tension, where nodes hosting tense actin filaments were pulled bidirectionally around the ring. Myo2 and Myp2 chaperoned self-assembling components into the ring organization, and anchored the ring against bridging instabilities. Thus, beyond force production, Myo2 and Myp2 are the principal organizers, bundlers and anchors of the contractile ring.
\end{abstract}




\section{Introduction}

In the late 1960s it emerged that force-generating actomyosin contractile machinery is not confined to muscle, with Schroeder's discovery of the contractile ring whose constriction divides cells during cytokinesis (Schroeder, 1968). With $\sim 5 \times 10^{11}$ daily sequences of ring assembly, maturation and constriction in humans (Ross, 1998), this machinery enables cell proliferation and the propagation of genetic material with extraordinary fidelity (Glotzer, 2017; Normand and King, 2010; Pollard and O'Shaughnessy, 2019). However, the relation between organization and force is far less clear for the contractile ring than for striated muscle, whose highly ordered organization is based on the sarcomere repeat unit. Sarcomeres contract when parallel actin filaments engage and slide past Myosin II thick filaments (Huxley and Hanson, 1954).

How tensile force emerges from the irregular actomyosin ring organization remains an open question. Following the discovery of actin filaments (Schroeder, 1972) and Myosin II (Fujiwara and Pollard, 1976) in the ring, the parallels with muscle led Schroeder and others to propose a muscle-like sliding filament mechanism (Fujiwara and Pollard, 1976; Mabuchi and Okuno, 1977; Schroeder, 1972). However, cytokinetic rings lack the ordered sarcomeric architecture of muscle (Laplante et al., 2016; Reymann et al., 2016).

In recent years super-resolution microscopies have probed the contractile ring organization in unprecedented nanoscale detail (Beach et al., 2014; Bellingham-Johnstun et al., 2021; Laplante et al., 2016). FPALM imaging showed that constricting fission yeast contractile rings, uniquely well characterized for their components and amounts (Courtemanche et al., 2016; Friend et al., 2018; Wu and Pollard, 2005), have an organization based on protein complexes called nodes (Laplante et al., 2016). This finding suggests a major shift from the sarcomeric paradigm of muscle. Each node includes $~ 8$ myosin II Myo2 dimers with plasma membrane-anchored tails, and the actin filament nucleator formin $\mathrm{Cdc} 12$, so actin filaments presumably emanate from nodes. With 200 nodes distributed irregularly around the ring at constriction onset, the node is the building block of the contractile ring, not the sarcomere. How a disordered node-based organization generates contractile ring tension is not established.

A major obstacle to finding the relationship between organization and tension production has been the near absence of ring tension measurements. Classic studies reported net forces $\sim 10$ $60 \mathrm{nN}$ in the furrows of echinoderm eggs (Hiramoto, 1970; Rappaport, 1967, 1977; Yoneda and Dan, 1972), but these include force from contiguous actomyosin cortex, or unverified assumptions 
were invoked. More recently, we measured $\sim 400 \mathrm{pN}$ tensions in contractile rings of fission yeast protoplasts, live cells with the cell wall removed (Stachowiak et al., 2014). Given the paucity of ring tension data, constriction rates are typically used as a proxy (Laplante et al., 2015; Piekny and Mains, 2002).

Actomyosin organizations typically use several myosin II isoforms with complementary roles. The fission yeast contractile ring has a second isoform, the unconventional myosin II Myp2, not belonging to the nodes and likely unanchored from the membrane (Bezanilla et al., 1997). The respective roles of Myo2 and Myp2 are not known. Animal cells express multiple Myosin II isoforms (NMIIA,B and C), with the more dynamic NMIIA mediating rapid contraction, while NMIIB promotes isometric tension (Shutova and Svitkina, 2018). In contractile rings NMIIA and NMIIB assemble into stacked minifilaments (Beach et al., 2014) and furrow ingression is slower in NMIIA-knockout cells (Yamamoto et al., 2019).

Here we study the tension mechanism in the node-based organization of the fission yeast cytokinetic ring. Measurements of ring tension in live cells and mutants of the myosin II isoforms show that both isoforms contribute, with a 2-fold greater contribution from Myo2. In simulations implementing the experimentally measured node organization, myosin generated tension by pulling node-attached actin filaments, explaining the observed bidirectional node motions around the ring (Laplante et al., 2016). Incoming components continuously self-assembled into the ring organization, chaperoned by Myo2 and Myp2 which maintain a dense actomyosin core and a dilute outer corona, respectively. Myo2 is the principal anchoring agent opposing bridging instabilities, the major threat to contractile ring integrity, and simulations reproduced bridging seen experimentally in the myo2-E1 mutant with weak actin binding (Lord and Pollard, 2004). Thus, beyond exerting force that makes ring tension, Myo2 and Myp2 have complimentary roles as the principal organizers, bundlers and anchoring agents of the contractile ring.

\section{Results}

\section{Method to measure contractile ring tension in fission yeast}

Past efforts to measure contractile ring tension in animal cells were complicated by interference from the actomyosin cortex. Here we used fission yeast protoplasts, living cells with enzymatically digested cell walls (Mishra et al., 2012). As fission yeast lacks an actin cortex, contractile rings in protoplasts are isolated so ring tension is opposed only by plasma membrane tension (Stachowiak 
et al., 2014).

The $\sim 5-7 \mu \mathrm{m}$ diameter spherical protoplasts were osmotically stabilized by $0.8 \mathrm{M}$ sorbitol, and $<5 \%$ had contractile rings which slid along the membrane as they constricted, their tension furrowing the membrane (Figure S1). Micropipet Aspiration measured the membrane tension in one lobe, $\sigma_{1}$, and Laplace's law yielded the tension in the unaspirated lobe, $\sigma_{2}=\sigma_{1} R_{2} / R_{1}$, where $R_{1}, R_{2}$ are the lobe radii (Figure $1 \mathrm{~B}$ and STAR Methods). The ring tension $T$ follows from a force balance, $T / R_{\text {ring }}=\sigma_{1} \cos \theta_{1}+\sigma_{2} \cos \theta_{2}$, where $R_{\text {ring }}$ is the ring radius and $\theta_{1}$ and $\theta_{2}$ the furrow angles. Angles and lobe radii were measured from images of the lobes with fluorescently labeled membrane. This improves our earlier method where membrane tension measured in interphase cells was used in the force balance in mitotic cells (Stachowiak et al., 2014). Membrane tensions were far lower in cells without rings (Figure S1A), so the previous method underestimated ring tensions.

Corroborating the method's assumption of a spatially uniform plasma membrane tension, the lobes flanking ring-induced furrows were almost perfect spherical caps. Membrane tension scaled approximately as the aspirated lobe radius $(p<0.01$, Figure 1D), indicating consistent osmotic pressures among cells $(\Delta P=440 \pm 30 \mathrm{~Pa}$, mean $\pm \mathrm{SD}, n=31$ cells, Figure $1 \mathrm{E})$.

\section{Contractile ring tension increases from $\sim 400$ to $\sim 800 \mathrm{pN}$ as the ring constricts}

We first used the method to measure the evolution of contractile ring tension as rings constricted. In measurements on 31 protoplast cells ring tensions increased as constriction progressed, from 400 to $\sim 800 \mathrm{pN}$ (Figure 2) with a mean of $640 \pm 290 \mathrm{pN}(\mathrm{SD}, n=31)$.

Each tension in Figure 2B refers to a different ring at a different stage of constriction. We also tracked successive tensions in five rings as they constricted. The tensions increased at $\leq 5 \%$ per min (Figure S2). Given a 50 min constriction time in protoplasts (Stachowiak et al., 2014), these rates are consistent with the single snapshots, for which tensions varied $\sim 2$-fold over the full constriction range.

\section{Contractile ring tension is $\mathbf{3 0 - 4 0 \%}$ lower in cells lacking Myp2p}

We measured the tension contributions of the two myosin II isoforms, Myo2 and Myp2, using protoplasts of cells with mutations of each myosin gene (Figure 2C). In protoplasts generated from $\Delta m y p 2$ cells lacking unconventional myosin II Myp2, the mean ring tension in 18 cells with rings 
at various stages of constriction was $400 \pm 230 \mathrm{pN}($ mean $\pm \mathrm{SD}), \sim 38 \%$ below the wild-type (WT) mean of $640 \mathrm{pN}$ (Figure 2D).

Compared at the same degree of constriction, the $\Delta m y p 2$ ring tensions were $\sim 5 \%-60 \%$ reduced from WT (Figures 2E \& 2F). Averaged over all polar angles $\phi\left(0^{0}<\phi<60^{0}\right)$, the reduction factor was $0.73 \pm 0.19$ (mean $\pm \mathrm{SD}, n=5$ bins), or a mean $\sim 27 \%$ tension loss (Figure 2I). These results suggest Myp2 contributes 30-40\% of the total WT ring tension.

\section{Contractile ring tension is $\sim 60 \%$ lower in the absence of Myo2 ATPase activity}

The temperature-sensitive myo2-E1 mutation (Balasubramanian et al., 1998) lacks ATPase activity (Lord and Pollard, 2004). The mean ring tension in 10 myo2-E1 protoplasts was $220 \pm 200 \mathrm{pN}$ $($ mean $\pm \mathrm{SD}), \sim 66 \%$ below the WT tension (Figure 2D). Rings constricted through a smaller range of angles $\left(0^{0}<\phi<45^{0}\right)$ than WT cells. Compared at the same degrees of constriction, tensions in myo2-E1 protoplasts were $\sim 44 \% \pm 19 \%$ of WT values (mean $\pm \mathrm{SD}, n=4$ bins) (Figures $3 \mathrm{E}, 3 \mathrm{~F}$ \& $3 \mathrm{G})$.

Thus, Myo2 accounts for $\sim 60 \%$ of the total WT ring tension. Together with the Myp2 contribution, the two myosin II isoforms appear to generate most or all contractile ring tension.

\section{Molecularly detailed simulation of the $S$. pombe cytokinetic ring}

We built a molecularly explicit mathematical model tightly constrained by experiment (Figure 3AC, Table S1, STAR Methods) to study how tension emerges from the node-based organization in the fission yeast ring. Our previous 2D fixed ring length model featured independently membraneanchored formin Cdc12 and Myo2 (Stachowiak et al., 2014). The 3D model developed here implements the measured node-based organization, incorporates Myp2 and evolves the length of the ring as it constricts. For details of the model, simulation method and parameters see STAR Methods and Table S1.

Node organization. The simulations implement the local organization revealed by super resolution microscopy, which showed Myo2, formin Cdc12, the IQGAP Rng2p and the F-BAR protein Cdc15p colocalizing in membrane-anchored nodes (Laplante et al., 2016) (Figure 3A). In simulations: (i) Near the membrane, a sphere represents Cdc15p and IQGAP Rng2p, with size and location matching the experimental $70 \mathrm{~nm}$ wide Cdc15p distribution. (ii) Formin Cdc12 dimers nucleate and grow actin filaments, binding the node $44 \mathrm{~nm}$ from the membrane. (iii) An ellipsoid 
with the experimental $132 \times 102 \times 102 \mathrm{~nm}$ dimensions located $94 \mathrm{~nm}$ from the membrane represents the heads of the $\sim 8$ Myo2 dimers.

Other components. Semiflexible actin filaments are represented by chains of rods with bending stiffness corresponding to the $\sim 10 \mu \mathrm{m}$ persistence length (Ott et al., 1993). The unconventional Myosin II, Myp2, forms puncta in constricting rings suggestive of clustering (Takaine et al., 2015), does not belong to nodes and is likely not directly anchored to the plasma membrane (see Discussion). We simulated Myp2 as unanchored $200 \mathrm{~nm}$ clusters of 16 molecules (Figure 3B), the values best reproducing experimental ring tensions and organization (Figure S3). The model did not include type $\mathrm{V}$ myosin Myo51, which dissociates before constriction completion (Laplante et al., 2015), or formin For3 since cytokinesis appears normal in $\Delta$ for 3 cells, including normal actin levels in rings (Feierbach and Chang, 2001).

Actin-myosin forces. Filaments intersecting Myo2 or Myp2 clusters bind and are pulled parallel to the filament (Figure 3B). As the filament unbinding forces are unknown, we scanned values and selected those best reproducing the experimental radial Myo2-Myp2 separation and actin bundle thickness (Figure S4). We used linear force-velocity relations for Myo2 and Myp2, with stall forces per cluster, $f_{\mathrm{MyO} 2}^{\text {stall }}$ and $f_{\mathrm{Myp} 2}^{\text {stall }}$, best reproducing our experimental ring tensions (Figure S5), and with the experimentally measured load-free Myo2 velocity $v_{m y o}^{0}$ for the estimated 25 myosin II heads per actin filament, $v_{m y o}^{0}=0.24 \mu \mathrm{m} \mathrm{s}^{-1}$ (Stark et al., 2010). The same loadfree velocity was used for Myp2, as Myo2 and Myp2 heads appear similar (Bezanilla and Pollard, 2000). The cluster force is evenly distributed over all bound filaments.

Other forces. Drag forces resist node membrane anchor motion parallel to the membrane, with a drag coefficient chosen to reproduce the experimental node velocity, $22 \pm 10 \mathrm{~nm} \mathrm{~s}^{-1}$ (Laplante et al., 2016). Node-node, Myp2-Myp2, node-Myp2 and actin-actin steric forces oppose overlapping of clusters or filaments (thus filaments cannot cross). Actin filaments are dynamically crosslinked by Ain $1 \mathrm{p} \alpha$-actinin dimers, the ring's most abundant passive crosslinker $(\sim 250$ at constriction onset) (Wu and Pollard, 2005) .

Amounts of ring components and turnover. The component amounts follow the experimental values throughout constriction (Courtemanche et al., 2016; Wu and Pollard, 2005), e.g. at onset simulated rings contain 3300 Myo2 molecules and 230 formin dimers in 210 nodes, 2300 Myp2 molecules in $~ 140$ clusters, and 230 actin filaments of total length $\sim 580 \mu \mathrm{m}$. Nodes stochastically bind a $0.2 \mu \mathrm{m}$ wide zone, representing the ingrowing septum edge (Cortes et al., 
2007), and unbind after a mean of 41 s consistent with reported component dissociation times (see STAR Methods). On binding a node, a formin nucleates and grows an actin filament that is stochastically shortened by cofilin-mediated severing (Elam et al., 2013). Results were insensitive to the nucleated filament orientation distribution. Myp2 clusters bind actin filaments with turnover time 46 s (Takaine et al., 2015). Rates of binding, actin polymerization and actin filament severing are set by demanding simulations reproduce the experimental densities of Myo2, Myp2 and formin and mean actin length, all evolving as constriction progresses.

Running the simulation. A dynamic boundary condition represented the ingrowing septum whose diameter, initially $3.7 \mu \mathrm{m}$, decreased at $2.4 \mathrm{~nm} \mathrm{~s}^{-1}$ (Thiyagarajan et al., 2015) for a total constriction time $\sim 25 \mathrm{~min}$. The width of the contractile ring was self-determined by the simulation.

\section{Ring components self-organize into a functional contractile ring}

Remarkably, in simulations of the model the incoming components self-organized into a contractile ring whose organization and tension reproduced experiment, independently of initial conditions (Figures 3-5). Even with an initially empty membrane, the stochastically incoming Myo2-containing nodes, Myp2 clusters, formins and freshly-nucleated actin filaments assembled without supervision into the same bundled actomyosin organization with the same tension, (Figures 3D \& S6).

Typical simulations used an initial condition with components randomly positioned in a $200 \mathrm{~nm}$ wide circular band, STAR methods. Before initiating constriction, the simulation was run for 3 mins until the ring self-organized. This dynamic organization maintained itself throughout constriction and reproduced many features measured in live cells (Figures 4E-G).

The actin filaments aligned into a circular bundle of mean thickness $131 \pm 4 \mathrm{~nm}$ (mean $\pm \mathrm{SD}, n=60$ rings at constriction onset) (Figure 3G), within the reported range from FPALM and cryoET, $\sim 100$ - 175 nm (Laplante et al., 2016; McDonald et al., 2017; Swulius et al., 2018). A fraction 9 \% of actin belonged to "whiskers" emanating from the bundle (Figures 3E and 3F) as observed in live cells (Vavylonis et al., 2008). A $100 \mathrm{~nm}$ thick Myo2 ring overlapped a concentric $200 \mathrm{~nm}$ thick inner Myp2 ring, both associated with the actin bundle (Figure 3G), quantitatively consistent with experiment (Laplante et al., 2015; McDonald et al., 2017). 


\section{Ring tension is generated by anchoring of actin barbed ends to the membrane}

Simulated contractile rings produced tensions close to the values we measured in live cells, including the tension increase throughout constriction (Figures 2 and 4A). Compared to the experimental mean $\sim 640 \mathrm{pN}$, the simulated mean was $\sim 740 \pm 170 \mathrm{pN}$ (Figure 4B), using best fit stall forces of $1.75 \mathrm{pN}$ (Myo2) and $1 \mathrm{pN}$ (Myp2) from a systematic scan. These lie in the range reported for smooth and skeletal muscle myosin II, $0.7-2.1 \mathrm{pN}$ (Ishijima et al., 1996; Tyska et al., 1999).

The tension mechanism relied on firm lateral anchoring of actin filament barbed ends to the plasma membrane (Stachowiak et al., 2014; Wang and O'Shaughnessy, 2019) via formins belonging to nodes (Alonso-Matilla et al., 2019; Thiyagarajan et al., 2017) (Figure 3A, B). Since myosin II tries to move toward barbed ends, this guaranteed filament tension (Figure 4C). Other hypothetical anchoring schemes generated less tension, or compression (Figure 4E). Both Myo2 and Myp2 contributed to tension; despite Myp2 being unanchored it also contributed, by pulling equally in both directions on the actin bundle of approximately zero net polarity (Stachowiak et al., 2012), Figure 4F.

This mechanism was apparent from the tension in individual actin filaments, $T_{\text {fil }}(x)$, which obeyed the relation for a barbed-end-anchored filament pulled by a density $c_{\text {bound }}$ of myosin II along its length, $T_{\text {fil }}(x)=c_{\text {bound }} \bar{f}_{\text {myo }} x$ ( $x$ is distance from the pointed end and $\bar{f}_{\text {myo }}$ the mean force per myosin II head) (Figure 4G and STAR methods).

The net ring tension $T$ sums the tensions of all filaments passing through a given crosssection (Figure 4D). We derived an approximate formula,

$$
T=\alpha\left[n f_{\mathrm{Myo} 2} \rho_{\text {Myo2 }}+m f_{\mathrm{Myp} 2} \rho_{\mathrm{Myp} 2}\right] l_{\mathrm{act}} / 2
$$

where a Myo2 node or Myp2 cluster contains $n=m=16$ heads, $\rho_{\text {Myo2 }}$ and $\rho_{\text {Myp2 }}$ are the cluster

densities along the ring, $f_{\mathrm{Myo} 2}, f_{\mathrm{Myp} 2}$ are the forces per head pulling actin filaments of mean length $l_{\text {act }}$, and the factor $\alpha$ accounts for whiskering, relative actin-myosin motion and the filament distribution width (see STAR Methods). Using experimental myosin densities and actin filament lengths (Courtemanche et al., 2016; Wu and Pollard, 2005) with no fitting parameters, Eq. 1 reproduced simulated and experimental tension profiles (Figure 4A).

\section{Membrane-anchored nodes move in both directions around the ring}


Due to this tension mechanism, nodes are slowly pulled clockwise or counterclockwise around the ring by myosin II (Figure 4C) as seen in live cells (Laplante et al., 2016). A node carries a mean of $\sim 1$ formin (Courtemanche et al., 2016; Wu and Pollard, 2005) and hence one filament pulled by myosin. Simulations accurately reproduced the experimental bimodal distribution of lateral node velocities in the membrane, (Figure $4 \mathrm{H})$.

Firm anchoring to the plasma membrane is required for this tension mechanism to work, so the actin filaments become tense when pulled by myosin II (Figure 4C). Indeed, this condition is satisfied: the mean node speed $22 \pm 11 \mathrm{~nm} \mathrm{~s}^{-1}$ ( $n=60$ rings, $10 \mathrm{~min}$ after constriction onset) is well below the load-free sliding velocity of myosin II $\sim 240 \mathrm{~nm} \mathrm{~s}^{-1}$ (Table S1). With artificially lowered node anchor drag coefficient, ring tension decreased $\sim 40 \%$ for drag extrapolated to zero, in accordance with a simple analytical model (Figure 4I and STAR methods). The residual tension is a second "fixed filament" contribution due to nodes hosting like-oriented filaments forming connected circular chains around the ring.

\section{Contractile rings have a dense actomyosin core and a dilute actomyosin corona}

Cross sections of simulated rings had two zones. At constriction onset the $\sim 130 \mathrm{~nm}$ thick actin bundle had $\sim 47$ filaments in its cross-section, in the range of 14-60 filaments reported from cryoET (Swulius et al., 2018). The bundle had a high-density central $100 \mathrm{~nm}$ core of $\sim 39$ filaments, surrounded by a more dilute corona of $\sim 5$-10 filaments filling a 3-fold greater area (Figure 5A). Myo2 was bound only to core actin filaments and the Myo2 cluster size defined the core diameter, while Myp2 bound all 47 filaments, spanning the entire bundle. Hence the myosins overlap radially, but the median Myp2 location is $26 \mathrm{~nm}$ further from the membrane than Myo2, as seen experimentally (Laplante et al., 2015; McDonald et al., 2017), (Figures 5A, B).

Thus, the core and corona are maintained primarily by Myo2 and Myp2, respectively. Indeed, in simulations with weakened Myo2-actin binding the dense core was abolished: most of the bundle unanchored from the membrane into straight Myp2-decorated bridges (Figures 5C (arrows) and S4). In simulations with weakened Myp2-actin binding the corona was lost, the core became less dense, and $\sim 1 / 2$ the ring's actin was in whiskers (Figures 5C, D and S4).

\section{Continuous reassembly maintains the organization and tension of the ring}


How is this contractile ring organization created and maintained? A long-standing question is how contractile rings remain functional as they constrict, given components are constantly shed (Schroeder, 1972; Wu and Pollard, 2005). Simulations revealed a mechanism of continuous reassembly: as rings constricted, dissociating components were continuously replaced by incoming components that self-assembled into the ring organization without supervision (Stachowiak et al., 2014). Self-assembly processes included myosin II-mediated zippering of formin-nucleated actin filaments into the bundle, entrainment of incoming nodes, meandering of growing filaments that welded the core and corona, and actin-dependent recruitment of Myp2 (Figures 3D \& 5E, F).

The continuous renewal of organization enabled rings to constrict without losing functionality. This mechanism required that the renewal time $(\sim 1 \mathrm{~min})$ be much less than the constriction time ( 25 mins), so ring components experienced almost no change in ring length before being replaced. Then the ring could rebuild itself at each progressively shorter length without disruption. Indeed, the organization was violently disrupted in simulations with constriction rates artificially boosted to exceed turnover rates. Nodes aggregated, rings buckled and the tension was dramatically lowered or negative (Figure $5 \mathrm{H}$ ).

The actomyosin bundle was self-sustaining: once initiated, it provided a platform for further zippering and for Myp2 to bind and participate in zippering-anchoring (Figure 3D). This history dependence enabled the ring to maintain a stable tension-generating organization, but also meant that radical organizational alterations were long lived. Artificially imposed partially unanchored bridges remained kinetically locked-in by Myp2-mediated zippering, without assistance from Myo2 (Figure 5G).

\section{Simulated myo2-E1 rings reproduce experimental tension loss and bridging defects}

Next we simulated the temperature-sensitive myo2-E1 mutant, which has $\sim 2 / 3$ reduced ring tensions (Figure 2) and severely disrupted organization with sections of the ring detaching from the membrane into straight bridges containing Myp2 (Laplante et al., 2015).

Myo2-E1 has minimal ATPase activity, binds actin weakly, and fails to translocate actin (Lord and Pollard, 2004; Stark et al., 2013). Accordingly, we simulated Myo2p-E1 with zero pulling force on actin, and a lower unbinding threshold (12 pN versus $40 \mathrm{pN}$ WT) which reproduced the experimental bridging phenotype. 
Simulations reproduced both features seen in live cells (Figure 6A). The mean tension $190 \pm 50 \mathrm{pN}$ was similar to the experimental mean of $220 \pm 200 \mathrm{pN}$ (Figure 6B) and tensions were $75 \%$ lower than simulated WT tensions, similar to the $\sim 60 \%$ reduction seen experimentally (Figure 6C). Due to the weakened Myo2-actin binding, centripetal Laplace forces pulled tense actin filaments away from Myo2 clusters, so entire ring sections formed straight actin bridges containing Myp2 only, anchored by a few tenuous attachments at bridge ends mediated by barbedend-anchored actin filaments (Figure 6A). Consequently, 10 mins into constriction the actin filament core was severely depleted, with $\sim 28 \%$ of actin in bridges or whiskers, 3 -fold more than in WT (Figure 6D).

\section{Simulated $\triangle m y p 2$ rings lack the corona and have reduced tension}

We simulated rings in $\triangle m y p 2$ cells by omitting Myp2 (Figure 6A). At constriction onset tensions were $\sim 50 \%$ the WT value, decreasing to $\sim 30 \%$ by the end of constriction (Figure 6C), with a mean of $330 \pm 60 \mathrm{pN}$, Figure 6B. By comparison, we measured a mean $\triangle m y p 2$ tension in live cells of $\sim 400 \pm 200 \mathrm{pN}, 65 \%$ of the WT value, Figure 6B.

In simulated $\triangle m y p 2$ rings the actin bundle had only a reduced density core of $\sim 27$ filaments (Figure 6A) and the corona was absent, as expected since Myp2 served as its scaffold in WT rings (Figure 5A,B). About 1/3 of actin filaments were chaotically oriented whiskers (Figure 6D).

The reduced ring tension was due in part to the absence of Myp2 pulling forces, and in part to the large whisker population which lowered the mean length of functional actin filaments belonging to the bundle. For the same amount of myosin II, shorter filaments produce lower tension (Eq. (1)).

\section{Discussion}

The contractile actomyosin ring generates tension for cell division, yet this most fundamental property has rarely been measured. In echinoderm embryos, Rappaport (Rappaport, 1967, 1977) and Hiramoto (Hiramoto, 1975) used deformation of microneedles and ferrofluid droplets, respectively, to measure inward radial furrow forces of $\sim 10-50 \mathrm{nN}$. However these were net forces, including opposing cortical forces. Yoneda and Dan estimated ring tensions of similar magnitude in dividing echinoderm eggs using an approximate force balance at the furrow (Yoneda 
and Dan, 1972) but the method assumed uniform cortical tension and a symmetrical cell shape with spherical caps, inconsistent with experiment (Hiramoto, 1967, 1975).

These historic studies were complicated by interference from contiguous actomyosin cortex. Here we isolated contractile rings in fission yeast protoplasts free of cortex or cell wall, so ring tension was balanced entirely by passive plasma membrane tension (Figure 1). We measured ring tensions $\sim 600 \mathrm{pN}$, increasing 2-fold as rings constricted, to which Myo2 and Myp2 contributed $\sim 60 \%$ and $\sim 30-40 \%$, respectively (Figure 2 ). Acting over a cross sectional area $\sim 0.01$

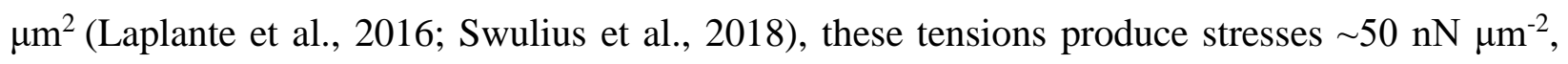
similar to the estimated $>8 \mathrm{nN} \mu \mathrm{m}^{-2}$ exerted by echinoderm egg rings (Rappaport, 1977). Thus, animal cell contractile rings, though much larger, may use similar tension-producing mechanisms.

\section{Constriction rate is not a proxy for ring tension}

In the absence of ring tension measurements, normal constriction rate is routinely taken to signify a normal contractile ring (Laplante et al., 2015; Piekny and Mains, 2002). We find this inference is unreliable. Rings in myo2-E1 cells constrict at the normal rate (Laplante et al., 2015) in spite of severe tension loss (Figure 2), while both tension and constriction rate (Laplante et al., 2015) are reduced in $\Delta m y p 2$ cells. Further, ring tension $T$ increased as rings constricted (Figure $2 \mathrm{~B}$ ), so the inward force $\sim T / R_{\text {ring }}$ increases dramatically with decreasing radius $R_{\text {ring }}$, yet constriction rates show no increase as constriction progresses (Thiyagarajan et al., 2015).

Thus, the constriction rate has no simple relation to ring tension. This is not surprising, given the tensions we measured are far too weak to oppose turgor pressure or to mechanically perturb the septum that grows inwards in the wake of the constricting ring (Thiyagarajan et al., 2015). The ring constriction rate is thus limited by the rate of growth of the septum to which it is indirectly attached. As septation is mediated by glucan synthases at the furrow (Cortes et al., 2007), our results suggest Myo2 affects their mean activity but Myp2 does not. If the glucan synthases are mechanosensitive, ring tension may regulate local septum growth rates to ensure proper septum closure (Pollard and O'Shaughnessy, 2019; Thiyagarajan et al., 2015).

\section{A sliding node mechanism generates contractile ring tension}


How does the irregular node-based organization of the contractile ring lead to tension? The critical feature is that nodes host actin filaments, whose barbed ends are thus firmly anchored to the membrane thanks to the small lateral node mobility in the membrane (Vavylonis et al., 2008). Thus when myosin II pulls actin filaments, host nodes slide slowly clockwise or counterclockwise around the ring and the filaments are made tense (Figure 7A). The myosin doing the pulling belongs to other nodes. Thus, in the disordered steady state ring, oppositely oriented nodes within range are continuously drawn together without ring shortening, which occurs on a much longer $25 \mathrm{~min}$ timescale. This is in contrast to sarcomere shortening, which shortens muscle.

Clockwise and counterclockwise node motions observed in live cells (Laplante et al., 2016) have a velocity distribution close to that predicted by simulations (Figure $4 \mathrm{H}$ ), with a mean speed well below the myosin II gliding velocity, consistent with substantial filament tensions (Table S1). A similar mechanism is used during ring assembly, when actin filaments are made tense as node pairs are pulled together from a broad band (Vavylonis et al., 2008). Constriction of partially anchored rings in fission yeast cell ghosts is quantitatively consistent with tension being generated by barbed-end anchoring of actin filaments to the membrane (Mishra et al., 2013; Wang and O'Shaughnessy, 2019).

The trends in contractile ring scaleup in larger animal cells suggest the broad mechanism based on barbed-end-anchored actin may be conserved. Ring length and width increase with cell size, but ring thickness remains several hundred nm, similar to fission yeast (O'Shaughnessy and Thiyagarajan, 2018). Thus, in larger cells the plasma membrane remains accessible for barbed end anchoring of actin filaments lying parallel to the plasma membrane.

\section{Myo2 and Myp2 make force, create ring organization and protect against instability}

In simulations Myo2 and Myp2 had broad, complimentary roles in the ring. Beyond force production, they helped create and protect the ring organization. The anchored nodes radially positioned Myo2 heads rather precisely, so actin and Myo2 cooperatively condensed into a dense core of $~ 30-35$ actin filaments with well-regulated spacing from the membrane (Figure 5A). This stable Myo2-bundled core allowed the unanchored Myp2 to roam beyond the range of Myo2 and entrain 5-10 more filaments in a loosely packed outer corona (Figures 5A \& 7B).

Myo2 also serves to anchor the actin bundle to the membrane, protecting the ring from bridging instabilities (Figure 7B). These are by far the biggest threat to contractile ring integrity, 
originating in the enormous energetic preference for a high tension curved bundle to shorten by straightening. In myo2-E1 cells with weakened actin binding, rings featured straight unanchored Myp2-containing bridges (Laplante et al., 2015). myo2-E1 simulations yielded a remarkably similar phenotype, with sections unanchoring into straight bridges (Figure 6A).

Why is Myo2 the ring's principal anchor? Depending on the duty ratio, up to 250 actinbound Myo2 heads $\mu^{-1}$ of ring (Wu and Pollard, 2005) oppose the Laplace forces $T / R \sim 220$ $\mathrm{pN} \mu \mathrm{m}^{-1}$. Duty ratios of NMIIA and NMIIB in animal cells are high under load (Kovacs et al., 2007), so Myo2 may exhibit similar mechanosensitivity. We estimate each Myo2 head must support $\sim 4 \mathrm{pN}$ (see STAR methods), within the $\sim 6-10 \mathrm{pN}$ unbinding threshold measured for muscle myosin II (Guo and Guilford, 2006; Nishizaka et al., 1995).

Furthermore, Myo2 is uniquely qualified as an anchor in the $S$. pombe ring. As a membrane-anchored motor protein using actin filaments for tracks, it can accommodate the constant elongation, fragmentation and sliding of filaments without unbinding. Passive actinbinding anchors would rapidly unbind from such dynamic filaments. Indeed, $\alpha$-actinin ain1 contributed insignificantly to bundling or ring tension (Figure S7) consistent with ainl deletion cells assembling and constricting rings normally (Wu et al., 2001) (see STAR methods).

More generally, the processivity of myosin may lend itself to unexpected anchoring roles. Anchoring of actin to the plasma membrane is thought accomplished by myosin VI in mammalian sensory hair cells (Altman et al., 2004; Self et al., 1999) and by myosin I during clathrin-mediated endocytosis in budding yeast (Pedersen and Drubin, 2019).

\section{Myo2 and Myp2 have complimentary roles in assembly of the constriction-ready ring}

To assemble the constriction-ready fission yeast ring, a precursor ring is first assembled from Myo2-containing nodes, followed by a $\sim 25$ min maturation phase when Myp2 is recruited (Wu and Pollard, 2005) (Figure 7C). We assumed Myp2 is not directly anchored to the membrane, suggested by several findings. Localization of Myp2 to the ring depends on actin filaments (Laplante et al., 2015; Takaine et al., 2015) and Myp2 lies >100 nm from the plasma membrane (McDonald et al., 2017), further than Myo2 (Laplante et al., 2015). Myp2 is present in unanchored straight bridges in mutants of Myo2 (Laplante et al., 2015) and in mutants of ADF/cofilin when Myo2 was shown to be left behind at the plasma membrane (Cheffings et al., 2019).

Why then is an unanchored myosin II recruited during maturation? Presumably, a major 
goal of maturation is to boost ring tension. This can be achieved by adding myosin or by elongating the actin filaments already present (Eq. (1)). Indeed, both measures are taken during maturation, when 2000 Myp2 molecules augment the 3000 Myo2 molecules in the precursor ring, and the polymerized actin increases $\sim 40 \%$ with no change in the number of $\mathrm{Cdc} 12$ formins (Courtemanche et al., 2016; Wu and Pollard, 2005). Using the best fit stall forces (Table S1) in eq. (1) gives $T \sim\left(\rho_{\text {myo2 }}+0.6 \rho_{\text {myp } 2}\right) l_{\text {act }}$, so these maturation measures increase ring tension $\sim 2$-fold.

However, elongating actin filaments in this fashion thickens the ring beyond the reach of Myo2, a significant technical challenge (Figure 7C). Unanchored Myp2 provides an ideal solution, able to roam from the membrane and bundle the additional actin into a corona while contributing to tension despite being unanchored.

Thus, the myosin II isoforms have complimentary roles during assembly of the ring into its constriction-ready form, just as they do during constriction. Membrane-anchored Myo2 is ideal for assembly of the nodes into a precursor ring, whereas unanchored myosin II would be useless for this task as nodes link up using just one or two filaments (Vavylonis et al., 2008). Once assembled, unanchored Myp2 is ideal to mediate thickening of the ring and a $\sim 2$-fold tension increase during maturation, a significant "beefing up."

\section{Acknowledgements}

This work was supported by National Institute of General Medical Sciences of the National Institutes of Health under award number R01GM086731 to B.O'S. and R01GM026132 to T.D.P. The content is solely the responsibility of the authors and does not necessarily represent the official views of the National Institutes of Health. H.F.C. is a Merck LSRF fellow. We thank C. Laplante and R. Arasada for yeast strains (all Pollard lab stock) and helpful discussions, J. Nikolaus for assistance with cell culture and the micromanipulator setup, and Dong An for assistance with figures.

\section{AUTHOR CONTRIBUTIONS}

B.O'S. conceived the study, B.O'S., T.D.P., E.K. and H.C. designed the experiments, B.O'S.,S.W., S.T., and Z.M. designed the model, H.C. performed the experiments, S.W., Z.M., and S.T. 
performed the simulations, B.O'S., H.C., S.W., Z.M., and S.T. analyzed the data. B.O'S, T.D.P., S.W., H.C, S.T., and Z.M. wrote the manuscript.

\section{COMPETING FINANCIAL INTERESTS}

The authors declare no competing financial interests. 


\section{References}

Alonso-Matilla, R., Thiyagarajan, S., and O'Shaughnessy, B. (2019). Sliding filament and fixed filament mechanisms contribute to ring tension in the cytokinetic contractile ring. Cytoskeleton (Hoboken) 76, 611-625.

Altman, D., Sweeney, H.L., and Spudich, J.A. (2004). The mechanism of myosin VI translocation and its load-induced anchoring. Cell 116, 737-749.

Balasubramanian, M.K., McCollum, D., Chang, L., Wong, K.C., Naqvi, N.I., He, X., Sazer, S., and Gould, K.L. (1998). Isolation and characterization of new fission yeast cytokinesis mutants. Genetics 149, 1265-1275.

Beach, J.R., Shao, L., Remmert, K., Li, D., Betzig, E., and Hammer, J.A., 3rd (2014). Nonmuscle myosin II isoforms coassemble in living cells. Curr Biol 24, 1160-1166.

Bellingham-Johnstun, K., Anders, E.C., Ravi, J., Bruinsma, C., and Laplante, C. (2021). Molecular organization of cytokinesis node predicts the constriction rate of the contractile ring. J Cell Biol 220.

Bezanilla, M., Forsburg, S.L., and Pollard, T.D. (1997). Identification of a second myosin-II in Schizosaccharomyces pombe: Myp2p is conditionally required for cytokinesis. Molecular Biology of the Cell 8, 2693-2705.

Bezanilla, M., and Pollard, T.D. (2000). Myosin-II tails confer unique functions in Schizosaccharomyces pombe: characterization of a novel myosin-II tail. Mol Biol Cell 11, 79-91.

Cheffings, T.H., Burroughs, N.J., and Balasubramanian, M.K. (2019). Actin turnover ensures uniform tension distribution during cytokinetic actomyosin ring contraction. Mol Biol Cell 30, 933-941.

Cortes, J.C., Konomi, M., Martins, I.M., Munoz, J., Moreno, M.B., Osumi, M., Duran, A., and Ribas, J.C. (2007). The (1,3)beta-D-glucan synthase subunit Bgs1p is responsible for the fission yeast primary septum formation. Mol Microbiol 65, 201-217.

Courtemanche, N., Pollard, T.D., and Chen, Q. (2016). Avoiding artefacts when counting polymerized actin in live cells with LifeAct fused to fluorescent proteins. Nat Cell Biol 18, 676-683.

Elam, W.A., Kang, H., and De la Cruz, E.M. (2013). Biophysics of actin filament severing by cofilin. FEBS Lett 587, 1215-1219.

Feierbach, B., and Chang, F. (2001). Roles of the fission yeast formin for3p in cell polarity, actin cable formation and symmetric cell division. Curr Biol 11, 1656-1665.

Friend, J.E., Sayyad, W.A., Arasada, R., McCormick, C.D., Heuser, J.E., and Pollard, T.D. (2018). Fission yeast Myo2: Molecular organization and diffusion in the cytoplasm. Cytoskeleton (Hoboken) 75, 164-173.

Fujiwara, K., and Pollard, T.D. (1976). Fluorescent antibody localization of myosin in the cytoplasm, cleavage furrow, and mitotic spindle of human cells. J Cell Biol 71, 848-875. Glotzer, M. (2017). Cytokinesis in Metazoa and Fungi. Cold Spring Harb Perspect Biol 9. Guo, B., and Guilford, W.H. (2006). Mechanics of actomyosin bonds in different nucleotide states are tuned to muscle contraction. Proc Natl Acad Sci U S A 103, 9844-9849.

Hiramoto, Y. (1967). Observations and measurements of sea urchin eggs with a centrifuge microscope. J Am Vet Med Assoc 150, 219-230.

Hiramoto, Y. (1970). Rheological properties of sea urchin eggs. Biorheology 6, 201-234. 
Hiramoto, Y. (1975). Force exerted by cleavage furrow of sea-urchin eggs. Dev Growth Differ 17, 27-38.

Huxley, H., and Hanson, J. (1954). Changes in the cross-striations of muscle during contraction and stretch and their structural interpretation. Nature 173, 973-976. Ishijima, A., Kojima, H., Higuchi, H., Harada, Y., Funatsu, T., and Yanagida, T. (1996). Multiple- and single-molecule analysis of the actomyosin motor by nanometer-piconewton manipulation with a microneedle: unitary steps and forces. Biophys J 70, 383-400. Kovacs, M., Thirumurugan, K., Knight, P.J., and Sellers, J.R. (2007). Load-dependent mechanism of nonmuscle myosin 2. Proc Natl Acad Sci U S A 104, 9994-9999.

Laplante, C., Berro, J., Karatekin, E., Hernandez-Leyva, A., Lee, R., and Pollard, T.D. (2015). Three Myosins Contribute Uniquely to the Assembly and Constriction of the Fission Yeast Cytokinetic Contractile Ring. Curr Biol 25, 1955-1965.

Laplante, C., Huang, F., Tebbs, I.R., Bewersdorf, J., and Pollard, T.D. (2016). Molecular organization of cytokinesis nodes and contractile rings by super-resolution fluorescence microscopy of live fission yeast. Proc Natl Acad Sci U S A 113, E5876-E5885.

Lord, M., and Pollard, T.D. (2004). UCS protein Rng3p activates actin filament gliding by fission yeast myosin-II. J Cell Biol 167, 315-325.

Mabuchi, I., and Okuno, M. (1977). The effect of myosin antibody on the division of starfish blastomeres. J Cell Biol 74, 251-263.

McDonald, N.A., Lind, A.L., Smith, S.E., Li, R., and Gould, K.L. (2017). Nanoscale architecture of the Schizosaccharomyces pombe contractile ring. Elife 6.

Mishra, M., Huang, Y., Srivastava, P., Srinivasan, R., Sevugan, M., Shlomovitz, R., Gov, N., Rao, M., and Balasubramanian, M. (2012). Cylindrical cellular geometry ensures fidelity of division site placement in fission yeast. J Cell Sci 125, 3850-3857.

Mishra, M., Kashiwazaki, J., Takagi, T., Srinivasan, R., Huang, Y., Balasubramanian, M.K., and Mabuchi, I. (2013). In vitro contraction of cytokinetic ring depends on myosin II but not on actin dynamics. Nat Cell Biol 15, 853-859.

Nishizaka, T., Miyata, H., Yoshikawa, H., Ishiwata, S., and Kinosita, K., Jr. (1995). Unbinding force of a single motor molecule of muscle measured using optical tweezers. Nature 377, 251-254.

Normand, G., and King, R.W. (2010). Understanding cytokinesis failure. Adv Exp Med Biol 676, 27-55.

0'Shaughnessy, B., and Thiyagarajan, S. (2018). Mechanisms of contractile ring tension production and constriction. Biophys Rev 10, 1667-1681.

Ott, A., Magnasco, M., Simon, A., and Libchaber, A. (1993). Measurement of the persistence length of polymerized actin using fluorescence microscopy. Phys Rev E Stat Phys Plasmas Fluids Relat Interdiscip Topics 48, R1642-R1645.

Pedersen, R.T.A., and Drubin, D.G. (2019). Type I myosins anchor actin assembly to the plasma membrane during clathrin-mediated endocytosis. J Cell Biol 218, 1138-1147. Piekny, A.J., and Mains, P.E. (2002). Rho-binding kinase (LET-502) and myosin phosphatase (MEL-11) regulate cytokinesis in the early Caenorhabditis elegans embryo. J Cell Sci 115, 2271-2282.

Pollard, T.D., and O'Shaughnessy, B. (2019). Molecular Mechanism of Cytokinesis. Annu Rev Biochem 88, 661-689.

Rappaport, R. (1967). Cell division - direct measurement of maximum yension exerted by furrow of echindoerm eggs. Sci 156, 1241-\&. 
Rappaport, R. (1977). Tensiometric studies of cytokinesis in cleaving sand dollar eggs. J Exp Zool 201, 375-378.

Reymann, A.C., Staniscia, F., Erzberger, A., Salbreux, G., and Grill, S.W. (2016). Cortical flow aligns actin filaments to form a furrow. Elife 5.

Ross, D.W. (1998). Multiple Steps in the Molecular Causes of Cancer. In Introduction to Oncogenes and Molecular Cancer Medicine (New York, NY: Springer New York), pp. 57-71. Schroeder, T.E. (1968). Cytokinesis: filaments in the cleavage furrow. Exp Cell Res 53, 272276.

Schroeder, T.E. (1972). The contractile ring. II. Determining its brief existence, volumetric changes, and vital role in cleaving Arbacia eggs. J Cell Biol 53, 419-434.

Self, T., Sobe, T., Copeland, N.G., Jenkins, N.A., Avraham, K.B., and Steel, K.P. (1999). Role of myosin VI in the differentiation of cochlear hair cells. Dev Biol 214, 331-341.

Shutova, M.S., and Svitkina, T.M. (2018). Mammalian nonmuscle myosin II comes in three flavors. Biochem Biophys Res Commun 506, 394-402.

Stachowiak, M.R., Laplante, C., Chin, H.F., Guirao, B., Karatekin, E., Pollard, T.D., and O'Shaughnessy, B. (2014). Mechanism of cytokinetic contractile ring constriction in fission yeast. Dev Cell 29, 547-561.

Stachowiak, M.R., McCall, P.M., Thoresen, T., Balcioglu, H.E., Kasiewicz, L., Gardel, M.L., and O'Shaughnessy, B. (2012). Self-organization of myosin II in reconstituted actomyosin bundles. Biophys J 103, 1265-1274.

Stark, B.C., James, M.L., Pollard, L.W., Sirotkin, V., and Lord, M. (2013). UCS protein Rng3p is essential for myosin-II motor activity during cytokinesis in fission yeast. PLoS One 8 , e79593.

Stark, B.C., Sladewski, T.E., Pollard, L.W., and Lord, M. (2010). Tropomyosin and myosin-II cellular levels promote actomyosin ring assembly in fission yeast. Mol Biol Cell 21, 989-

1000.

Swulius, M.T., Nguyen, L.T., Ladinsky, M.S., Ortega, D.R., Aich, S., Mishra, M., and Jensen, G.J. (2018). Structure of the fission yeast actomyosin ring during constriction. Proc Natl Acad Sci U S A 115, E1455-E1464.

Takaine, M., Numata, O., and Nakano, K. (2015). An actin-myosin-II interaction is involved in maintaining the contractile ring in fission yeast. J Cell Sci 128, 2903-2918.

Thiyagarajan, S., Munteanu, E.L., Arasada, R., Pollard, T.D., and O'Shaughnessy, B. (2015). The fission yeast cytokinetic contractile ring regulates septum shape and closure. J Cell Sci 128, 3672-3681.

Thiyagarajan, S., Wang, S., and O'Shaughnessy, B. (2017). A node organization in the actomyosin contractile ring generates tension and aids stability. Mol Biol Cell 28, 32863297.

Tyska, M.J., Dupuis, D.E., Guilford, W.H., Patlak, J.B., Waller, G.S., Trybus, K.M., Warshaw, D.M., and Lowey, S. (1999). Two heads of myosin are better than one for generating force and motion. Proc Natl Acad Sci U S A 96, 4402-4407.

Vavylonis, D., Wu, J.Q., Hao, S., O'Shaughnessy, B., and Pollard, T.D. (2008). Assembly mechanism of the contractile ring for cytokinesis by fission yeast. Science 319, 97-100. Wang, S., and O'Shaughnessy, B. (2019). Anchoring of actin to the plasma membrane enables tension production in the fission yeast cytokinetic ring. Mol Biol Cell 30, 20532064. 
Wu, J.Q., Bahler, J., and Pringle, J.R. (2001). Roles of a fimbrin and an alpha-actinin-like protein in fission yeast cell polarization and cytokinesis. Mol Biol Cell 12, 1061-1077. Wu, J.Q., and Pollard, T.D. (2005). Counting cytokinesis proteins globally and locally in fission yeast. Science 310, 310-314.

Yamamoto, K., Otomo, K., Nemoto, T., Ishihara, S., Haga, H., Nagasaki, A., Murakami, Y., and Takahashi, M. (2019). Differential contributions of nonmuscle myosin IIA and IIB to cytokinesis in human immortalized fibroblasts. Exp Cell Res 376, 67-76.

Yoneda, M., and Dan, K. (1972). Tension at the surface of the dividing sea-urchin egg. J Exp Biol 57, 575-587. 


\section{Figures}

A

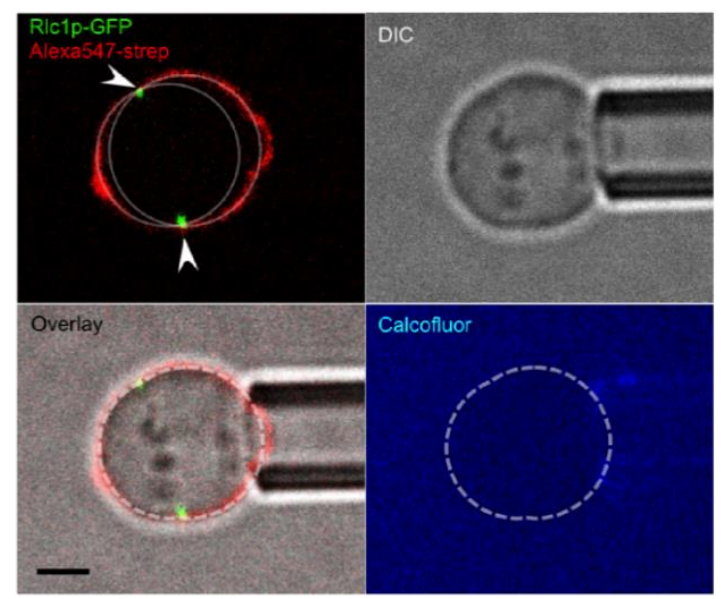

C

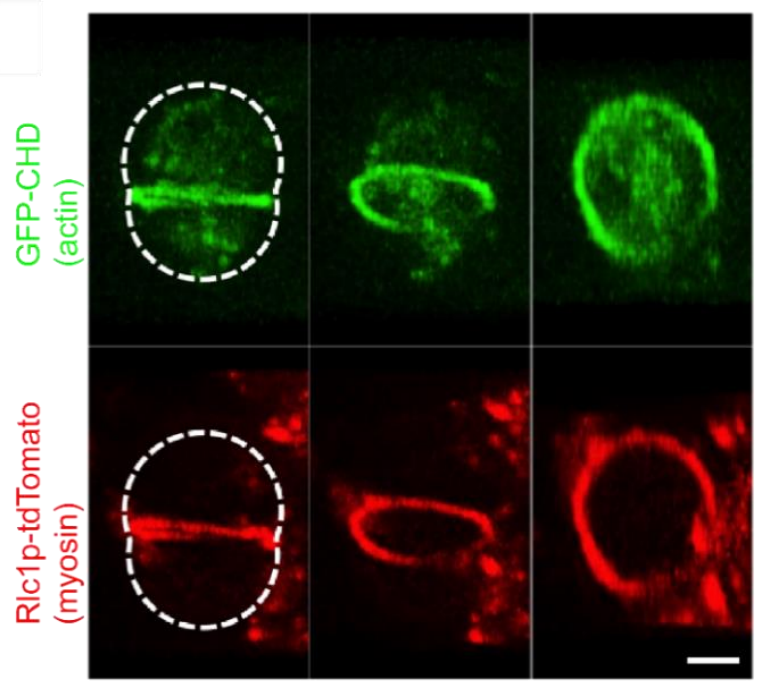

B
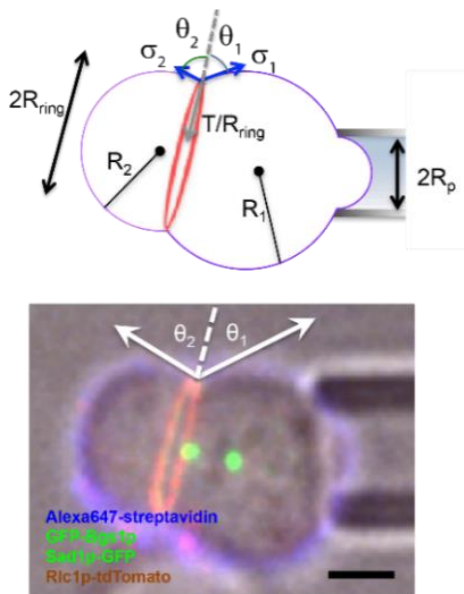

D

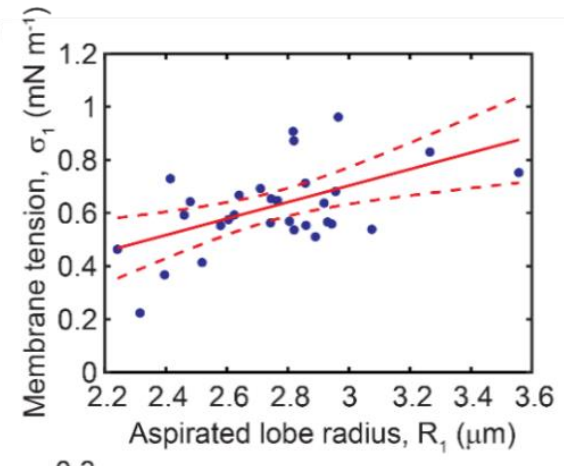

E

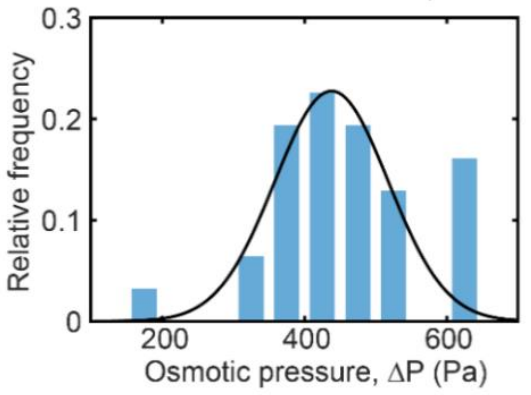

Figure 1. Method to measure cytokinetic ring tension in fission yeast protoplasts.

(A) A protoplast cell is held by an aspirating pipette and furrowed by a cytokinetic ring. The ring is marked by myosin II light chain (Rlc1p-3GFP), the plasma membrane by Alexa555streptavidin (red). The furrow geometry (arrowheads) was measured by fitting circles to the flanking lobes. Absence of calcofluor white staining indicates minimal regrowth of cell wall (lower right). Scale bars, $2 \mu \mathrm{m}$. 
(B) Top, geometry for ring tension measurements. Bottom, fluorescence and bright field overlay of an aspirated protoplast with labeled spindle pole bodies (Sad1p-GFP), myosin and Bgs1p in cytokinetic rings (Rlc1p-tdTomato, GFP-Bgs1p), and plasma membrane (Alexa647streptavidin).

(C) Confocal micrographs of a furrowed protoplast. Actin and myosin II colocalize in the cytokinetic ring, and actin is visible in the cell interior, but actomyosin cortex is absent. Images from left to right show $3 \mathrm{D}$ reconstructions consecutively rotated $40^{\circ}$. Dashed white line, cell boundary.

(D) Membrane tensions of furrowed protoplasts versus aspirated lobe radius, $\mathrm{R}_{1}(n=31)$. An approximately linear relation holds, with best fit slope $0.31 \pm 0.09 \mathrm{mN} / \mathrm{m} / \mu \mathrm{m}$ (red line, $\mathrm{F}$ test, $p$ $<0.01$, and Pearson correlation coefficient, $r=0.52$; dashed curves, 95\% confidence interval). (E) Histogram of osmotic pressures in furrowed protoplasts of (D), obtained from Laplace's Law $\left(\Delta P=2 \sigma_{1} / R_{1}\right)$. Mean pressure is $440 \pm 30 \mathrm{~Pa}($ mean $\pm \mathrm{SD})$, or osmolarity $\sim 0.2 \mathrm{mM}$. Solid curve: best fit Gaussian. 
A

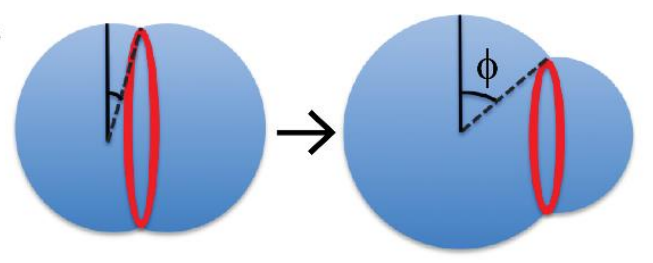

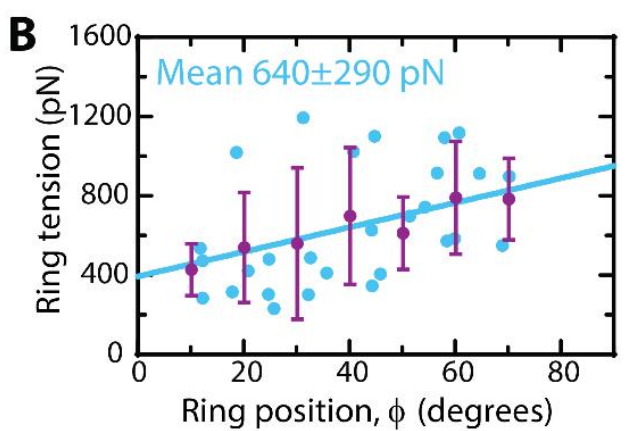

C

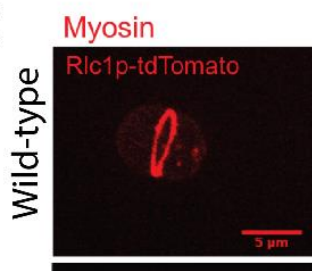

Membrane

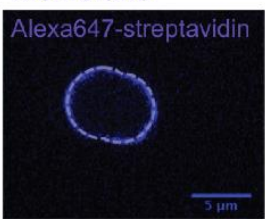

Brightfield
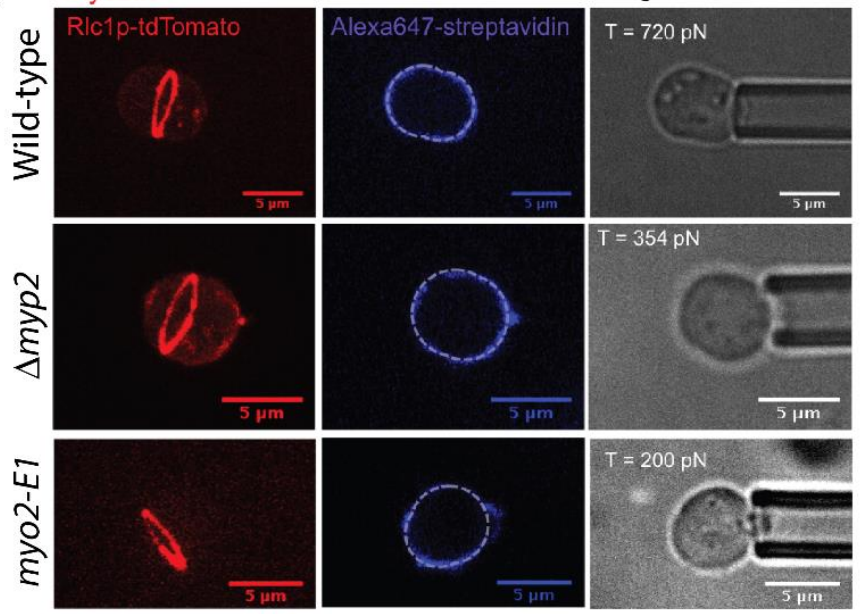

$\mathbf{E}$

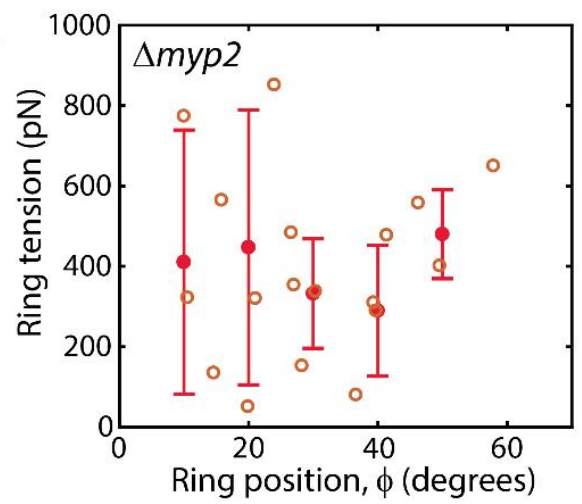

D

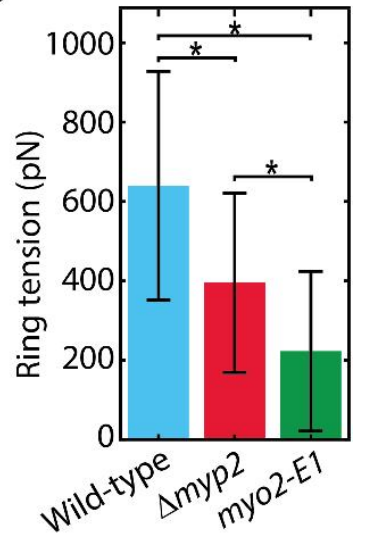

H
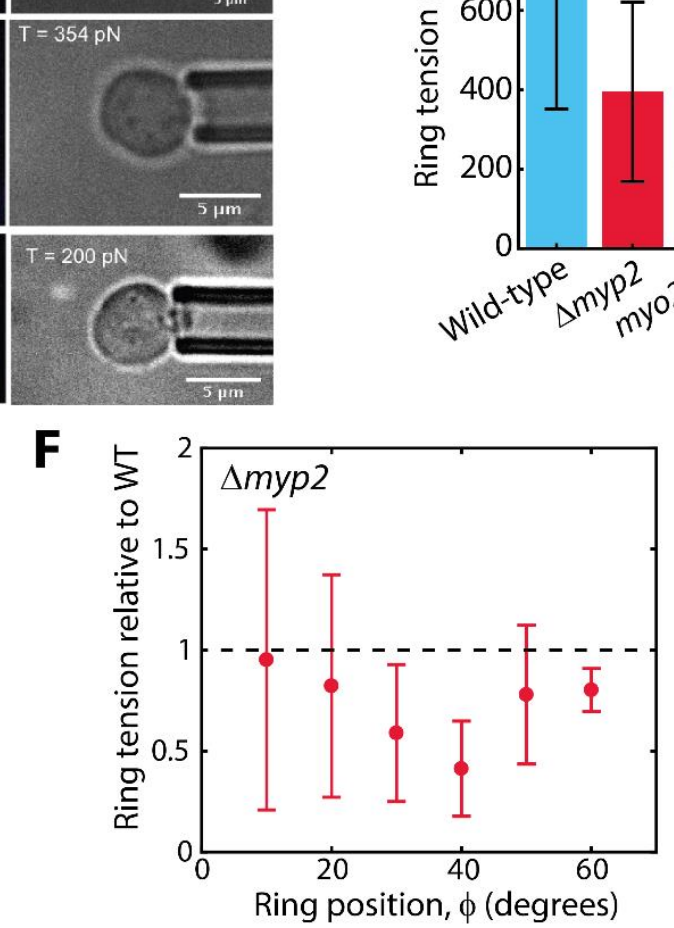

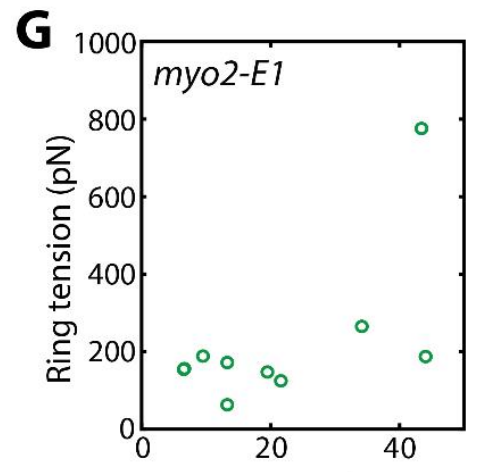

Ring position, $\phi$ (degrees)

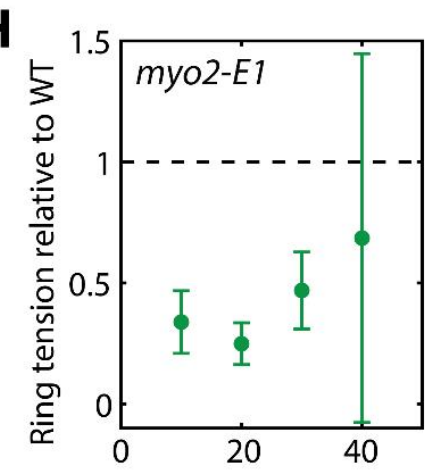

Ring position, $\phi$ (degrees)

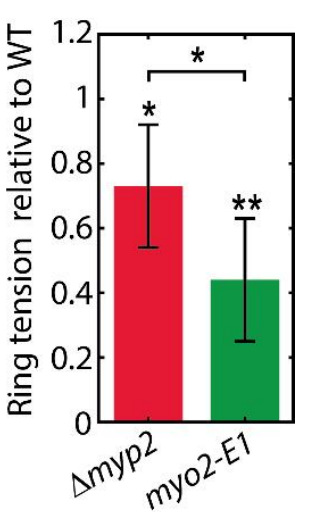


Figure 2. Cytokinetic ring tension increases as the ring constricts and is accounted for by the myosin II isoforms Myo2 and Myp2

(A) Schematic of a constricting ring in a protoplast, located by the angle $\phi$ between the ring and large lobe equatorial planes. As the ring constricts it slides along the inside of the protoplast membrane.

(B) Top, ring tensions versus ring location $\phi(n=31)$. Ring tension increases as the ring constricts (Pearson coefficient $r=0.40$; solid line, best linear fit). Purple, binned tensions (bin width, $10^{\circ}$ ). Best linear fit (solid line), $58 \pm 10 \mathrm{pN}$ per 10 degrees ( $p=0.0023, \mathrm{~F}$ test). Error bars, SD. See Figure S2 for tensions versus time for individual constricting rings.

(C) Confocal fluorescence and bright field images of WT, $\Delta m y p 2$ and myo2-E1 protoplasts aspirated to measure the tension of a contractile ring (left column). Each row shows images of the same cell. Plasma membrane, dashed lines (middle column). Measured ring tension indicated (right column). Table $\mathrm{S} 2$ lists the $S$. pombe strains used in this study.

(D) Ring tensions averaged over all ring positions for WT, $\triangle m y p 2$ and myo2-E1 protoplasts. Error bars, SD.

(E) Ring tensions in $\triangle m y p 2$ protoplasts (open circles, $n=18$ ), and binned tension values (closed circles, bin width $10^{\circ}$ ) versus ring location $\phi$. Error bars, SD.

(F) $\Delta m y p 2$ tension relative to WT tension versus ring location. For each bin of ring locations $\phi$ (bin width, $10^{\circ}$ ) the ratio of the binned $\triangle m y p 2$ tension in $(\mathbf{C})$ to the binned WT tension in Figure 2B is plotted. Error bars, SEM.

(G) Ring tensions in myo2-E1 protoplasts versus ring location $\phi(n=10)$.

(H) myo2-E1 tension relative to WT tension versus ring location. For each bin of ring locations the ratio of the binned myo2-E1 tension from (E) to the corresponding binned WT tension in Figure 2B is plotted. Error bars, SEM.

(I) Mean tension ratios for myo2-E1 and $\triangle m y p 2$ protoplasts shown in (D) and (F), averaged over angles $\phi$ (mean $\pm \mathrm{SD})$. ${ }^{*} p<0.05, * * \mathrm{p}<0.01$, Student's t test. 
A
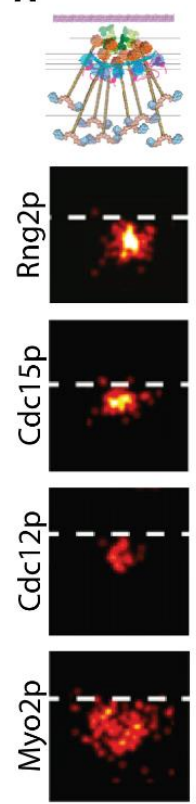
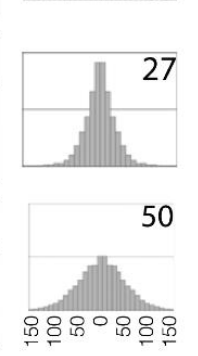

B

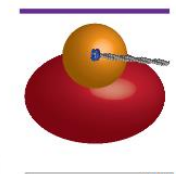

30
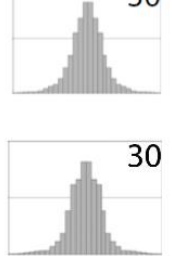

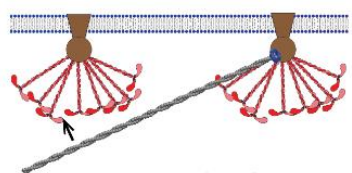

Myo2-actin binding

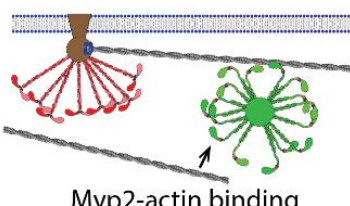

Myp2-actin binding

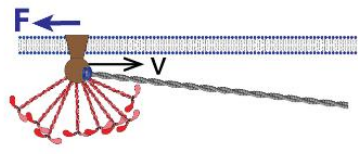

Anchor drag
Forces

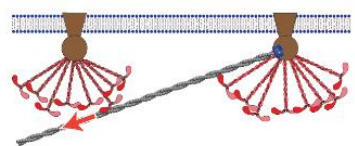

Myo2-actin pulling

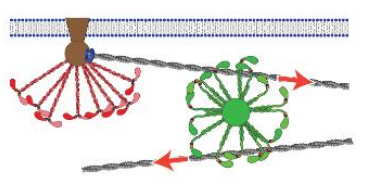

Myp2-actin pulling

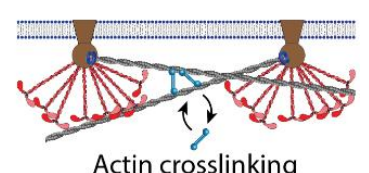

Actin crosslinking

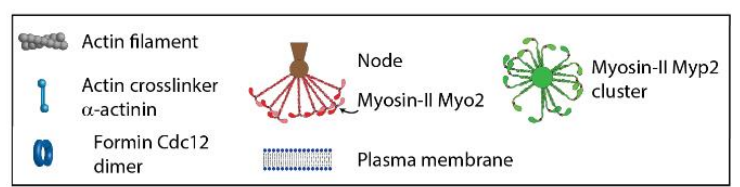

Turnover

C

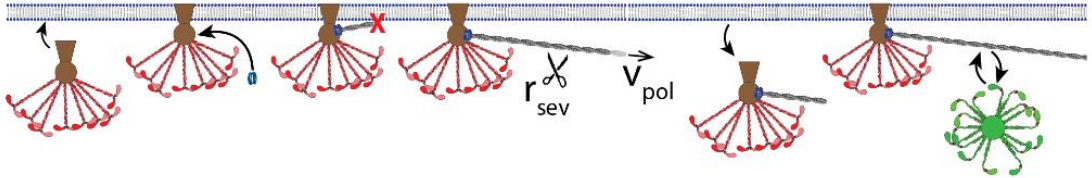

D

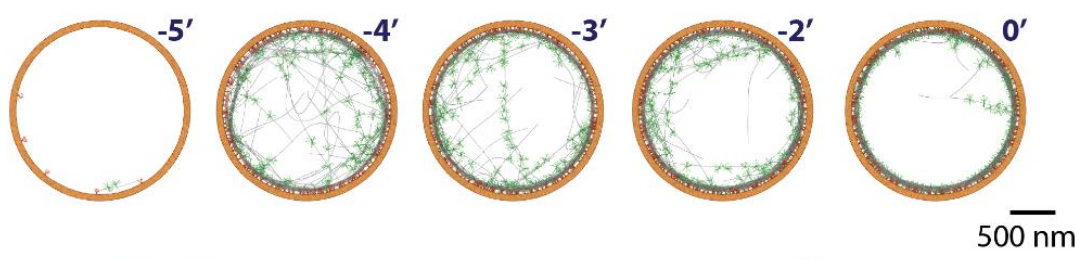

$\mathbf{E}$

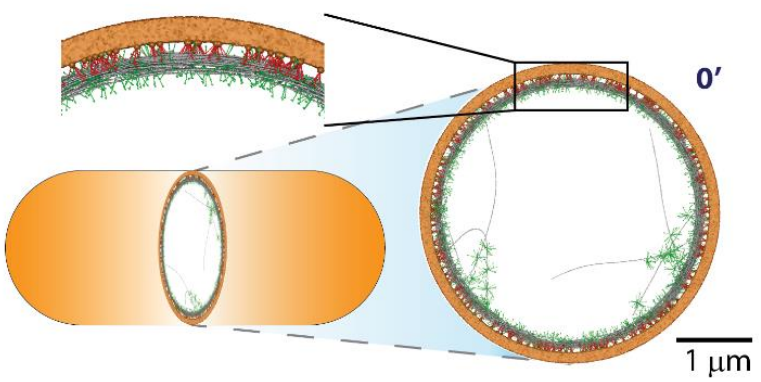

$\mathbf{F}$

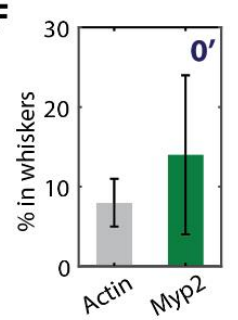

G
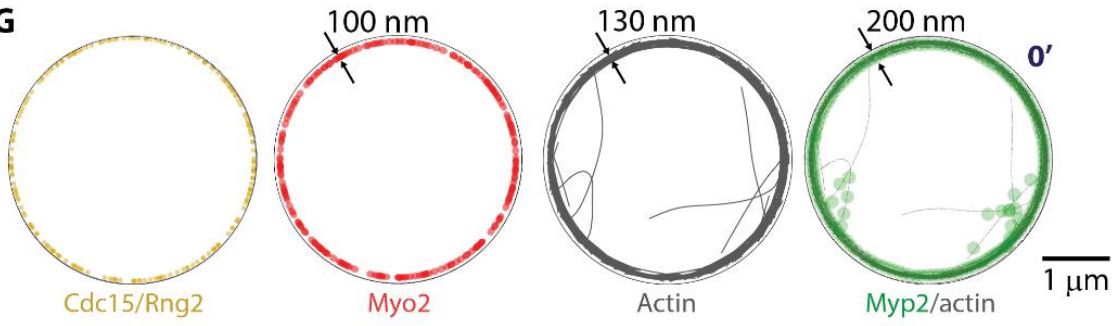

Figure 3. Mathematical model of the fission yeast contractile ring predicts self-assembly of components into a functional ring 
Color code used in this and subsequent figures: Myo2 (red); Myp2 (green); Actin (grey).

(A) The model implements the experimental node organization in constricting rings, including node stoichiometries and spatial distributions. Lower 4 rows, FPALM images of node

components (left) and radial density distributions (right; radii with $75 \%$ of FPALM localizations in nm; plasma membrane, dashed white line). Top row, schematic of membrane-anchored node organization (left) and coarse-grained model representation of Myo2 heads (red) and Rng2 and Cdc15 (brown). Formin Cdc12 dimers bind the node $40 \mathrm{~nm}$ from the membrane. FPALM images, density distributions and node schematic reproduced with permission from (Laplante et al., 2016).

(B) Forces in the model.

(C) Turnover. Nodes bind and dissociate from the plasma membrane. Formin Cdc12 dimers bind nodes and grow randomly oriented actin filaments at rate $v_{\text {pol }}$. Cofilin stochastically severs filaments. Myp2 clusters bind/unbind filaments.

(D) Starting from a bare membrane, ring components self-assemble into a functional actomyosin bundle within 5 mins (see Figure S6). Myo2 (red) and Myp2 (green) are rendered with explicit molecular detail for clarity.

(E) Simulated ring at constriction onset, following 3 min of equilibration from a random initial condition.

(F) Fraction of actin and Myp2 in whiskers at constriction onset (mean $\pm \mathrm{SD}, n=60$ rings).

(G) Spatial distributions of components in the steady state ring of (D). 

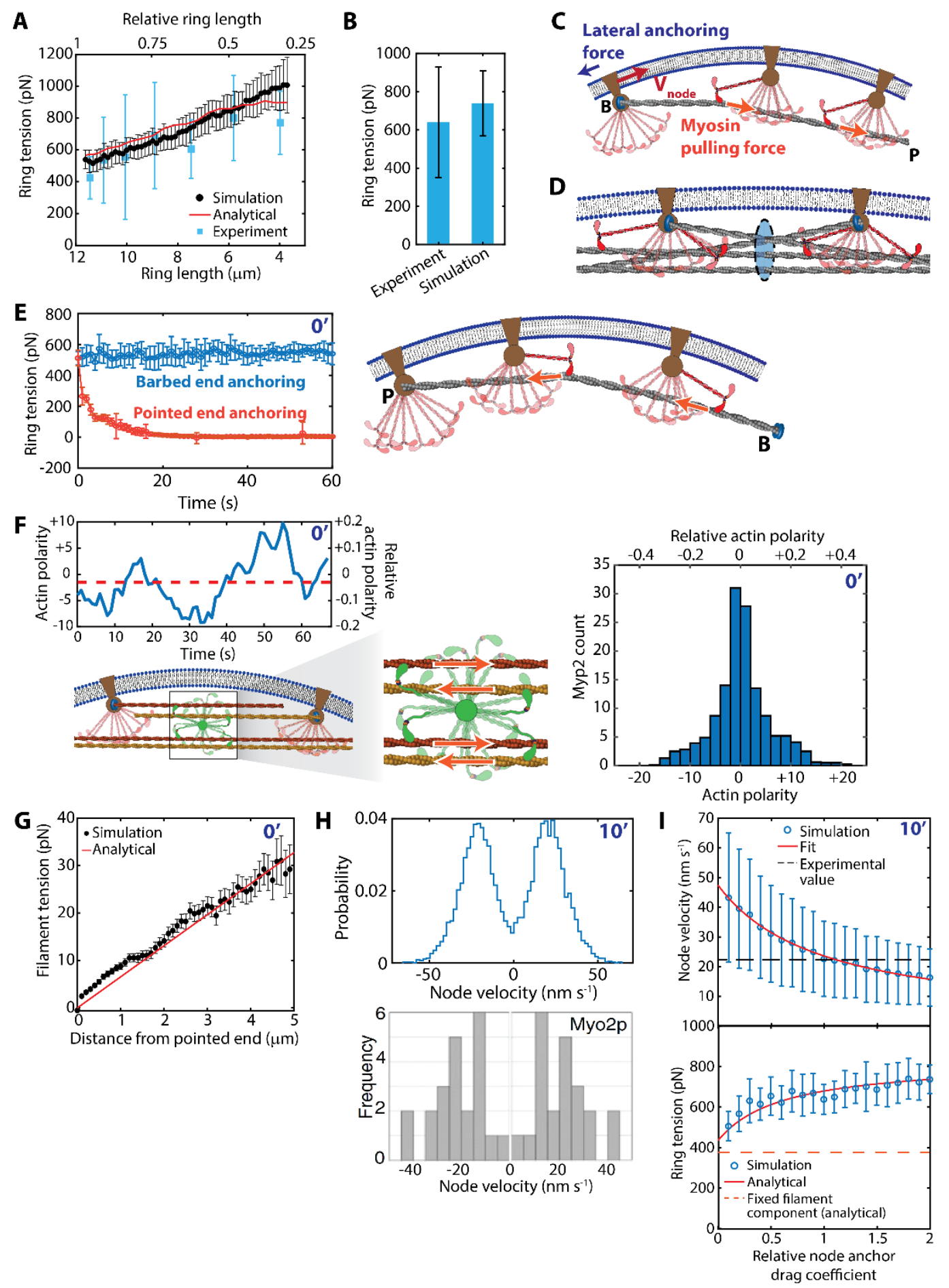

Figure 4. Sliding node ring tension mechanism

(A) Simulated ring tensions WT (black, mean $\pm \mathrm{SD}, \mathrm{n}=60$ simulations, parameters as in Table S1). WT experimental tensions (blue, data from Figure 2B, compared at same length relative to initial length). Approximate analytical expression, Equation (1) (red curve). 
(B) Mean WT tensions from experiment ( $n=31$, Figure 2B) and simulations ( $n=60$ simulations). Error bars are SD.

(C) Sliding node ring tension mechanism. On average a node anchors the barbed end of one actin filament to the membrane. Pulled by myosin, filament tension results since the node-membrane anchor drag force large enough that the node velocity $v_{\text {node }}$ is well below the load-free myosin II velocity.

(D) The ring tension sums tensions of all filaments passing through a given ring cross-section. Nodes with oppositely oriented actin filaments are drawn together.

(E) Pointed end anchoring fails to generate tension. Tension was lost within $\sim 30$ seconds (left, $n=20$, error bars are SD) as filaments are compressed or buckled by myosin II (right).

(F) Unanchored Myp2 clusters contribute to tension by pulling on equal numbers of oppositely oriented filaments. Net filament polarity bound to a Myp2 cluster is small and fluctuates in time (top left). Net polarity histogram for all Myp2 clusters in a contractile ring ( $\mathrm{n}=10$ rings at constriction onset, right). Relative polarities are normalized by the maximum value (all filaments having same polarity).

(G) Mean tension profile along one actin filament, constriction onset (mean \pm SEM, n = 174 filaments). Red line: expected linear profile for uniform myosin II binding (see main text and STAR Methods).

(H) Nodes move bidirectionally around the ring. Top: bimodal distribution of node velocities in simulated rings ( $n=60$ simulations, $\sim 120$ nodes per ring, 500-600 s after constriction onset). Nodes were tracked for 40s. Bottom: Myo2 velocity distribution measured in cells, reproduced from ref. (Laplante et al., 2016).

(I) Normal ring tension requires firm anchoring of actin filament barbed ends to the plasma membrane. Lowering node anchor drag coefficient decreases simulated ring tensions, approaching the drag-independent fixed filament component $(\mathrm{n}=10$ rings per data point, $10 \mathrm{~min}$ after constriction onset, error bars represent SD). The tension data are well described by an analytical model (red curve, STAR Methods). Anchor drags are relative to the WT simulated value (see main text). 

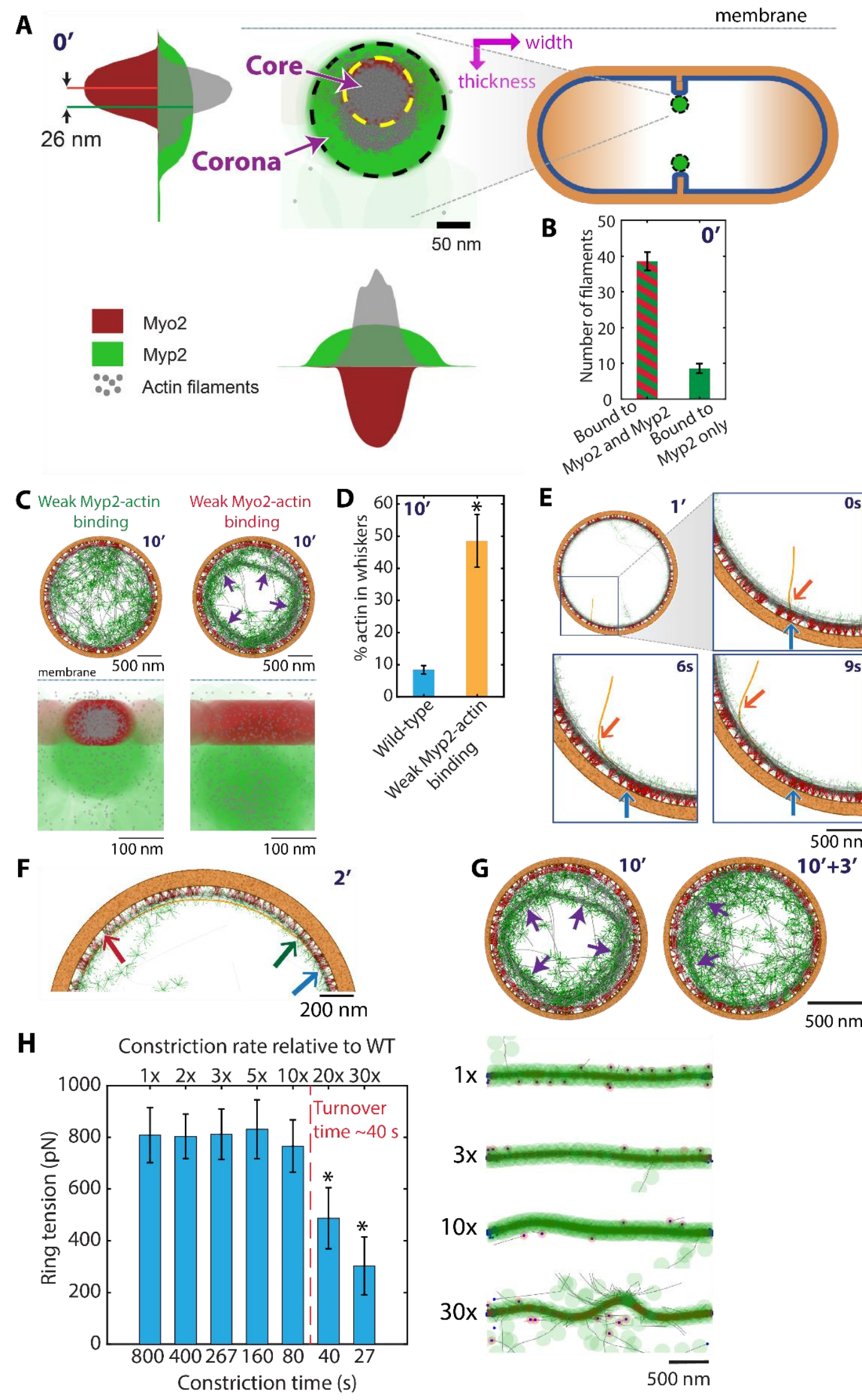


\section{Figure 5. Contractile rings have a dense actomyosin core and a dilute corona}

(A) Localizations of Myo2 heads, Myp2 heads and actin filaments in the cross-section of a ring at constriction onset. Projection of all cross-sections around the ring are shown. Myo2 and Myp2 organize a dense actin bundle core and a more dilute outer corona, respectively. Indicated core and corona boundaries are circles of radius the Myo2 ellipsoid or Myp2 cluster width, respectively, centered on the median Myo2 or Myp2 head location. Density profiles across the ring width and thickness are plotted (arbitrary units, same units for Myo2 and Myp2).

(B) Fraction of filaments bound to both Myo2 and Myp2 (core) or Myp2 only (corona). $\mathrm{n}=60$ rings 10 min after constriction onset, mean \pm SD.

(C) Weakening Myo2- or Myp2-actin binding leads to loss of the core or corona, respectively. The actin-Myp2 or actin-Myo2 filament-cluster unbinding threshold was lowered from $30 \mathrm{pN}$ to $10 \mathrm{pN}$ (left) or from $40 \mathrm{pN}$ to $10 \mathrm{pN}$ (right). Cross-sections are shown in the bottom row.

(D) In rings with weakened Myp2-actin binding (10 pN threshold) the fraction of actin in whiskers increased $\sim 6$-fold due to corona loss ( $\mathrm{n}=10$ rings, mean $\pm \mathrm{SD}$; $* p<0.05$ ).

(E) Myosin II-mediated zippering (orange arrow) of a newly nucleated actin filament, $1 \mathrm{~min}$ after constriction onset. The filament was nucleated by a formin (blue arrow), captured by Myp2, then entrained into the core by Myo2.

(F) An actin filament (orange) meandering between core and corona, 2 min after constriction onset. Core-corona transition points (green and red arrows) and barbed end (blue arrow) are indicated.

(G) Ring organizational defects can be long-lived. Using an initial condition with bridges (ring of panel (C)), after 3 min the organization is partially but not fully self-repaired (arrows).

(H) Functional constricting rings require component turnover to be faster than constriction. When constriction rates are artificially increased beyond the turnover rate, ring tension is reduced $(n=10$ rings, error bars $\mathrm{SD}, * \mathrm{p}<0.05)$ and organization drastically disrupted with buckled rings $\left(360^{\circ}\right.$ merged in-plane side-view of $1 \mu \mathrm{m}$ radius rings, right). Constriction time defined as $-R_{\text {ring }} /\left(d R_{\text {ring }} / d t\right)$. 
A
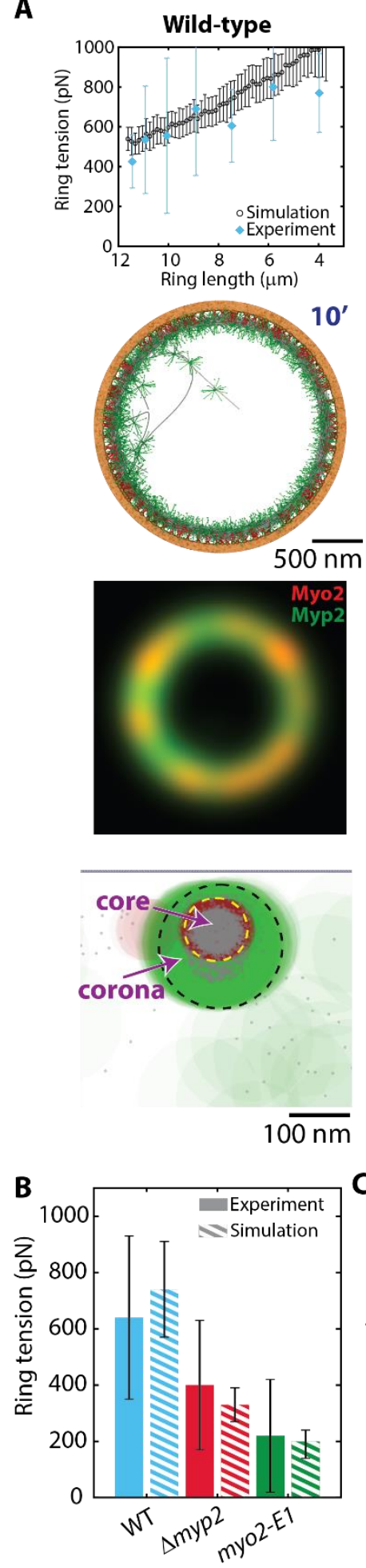
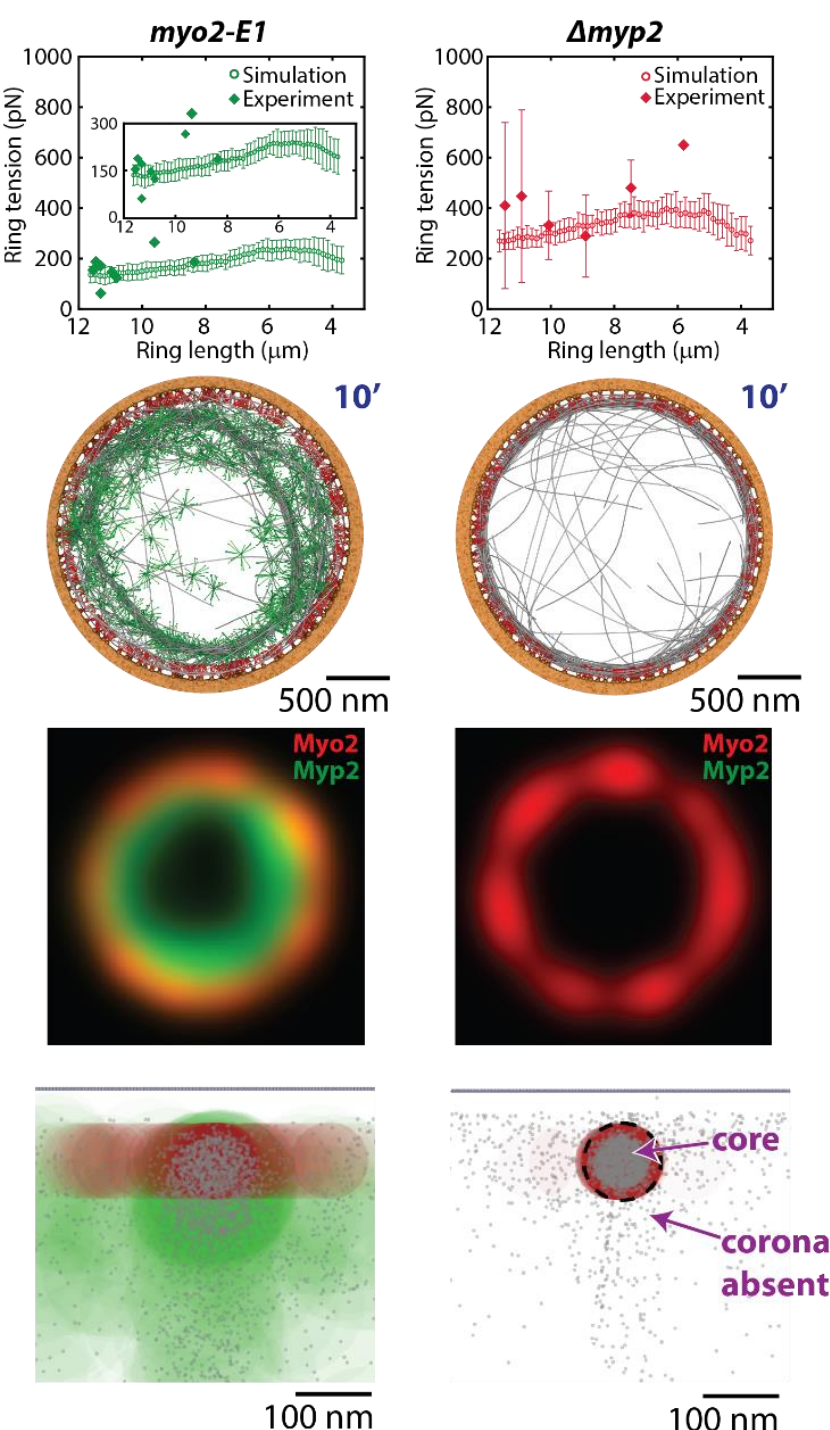

$10 \overline{\mathrm{nm}}$
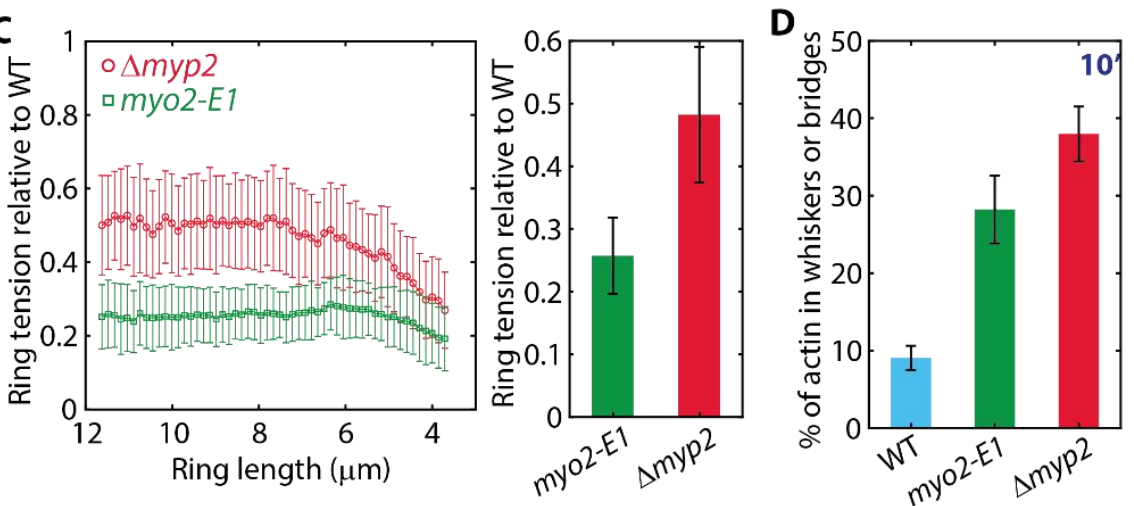
Figure 6. Simulations reproduce tension loss and organizational defects in contractile rings of myo2-E1 and $\triangle m y p 2$ mutants

(A) Ring tensions in simulations of contractile rings in WT, myo2-E1, and $\Delta m y p 2$ cells $(\mathrm{n}=60$

per simulation condition, mean $\pm \mathrm{SD}$ ) compared to the experimental tensions of Figure $2 \mathrm{C}$.

Simulation snapshots show bridging instabilities in myo2-E1 rings and loss of corona in $\Delta m y p 2$

rings (second row). Simulated fluorescence microscopy images (2D Gaussian point spread

function, width $310 \mathrm{~nm}$, third row) and cross-section component localizations (fourth row) of the rings shown in the second row.

(B) Mean simulated and experimental tensions of (A). Error bars are SD.

(C) Ring tensions of simulated myo2-E1 and $\triangle m y p 2$ rings of (A) relative to wild type simulated ring tensions of (A), versus ring length. Mean relative values over all lengths (right).

(D) Mean fraction of actin in whiskers or bridges in the simulated rings of (A) 10 min after constriction onset. 

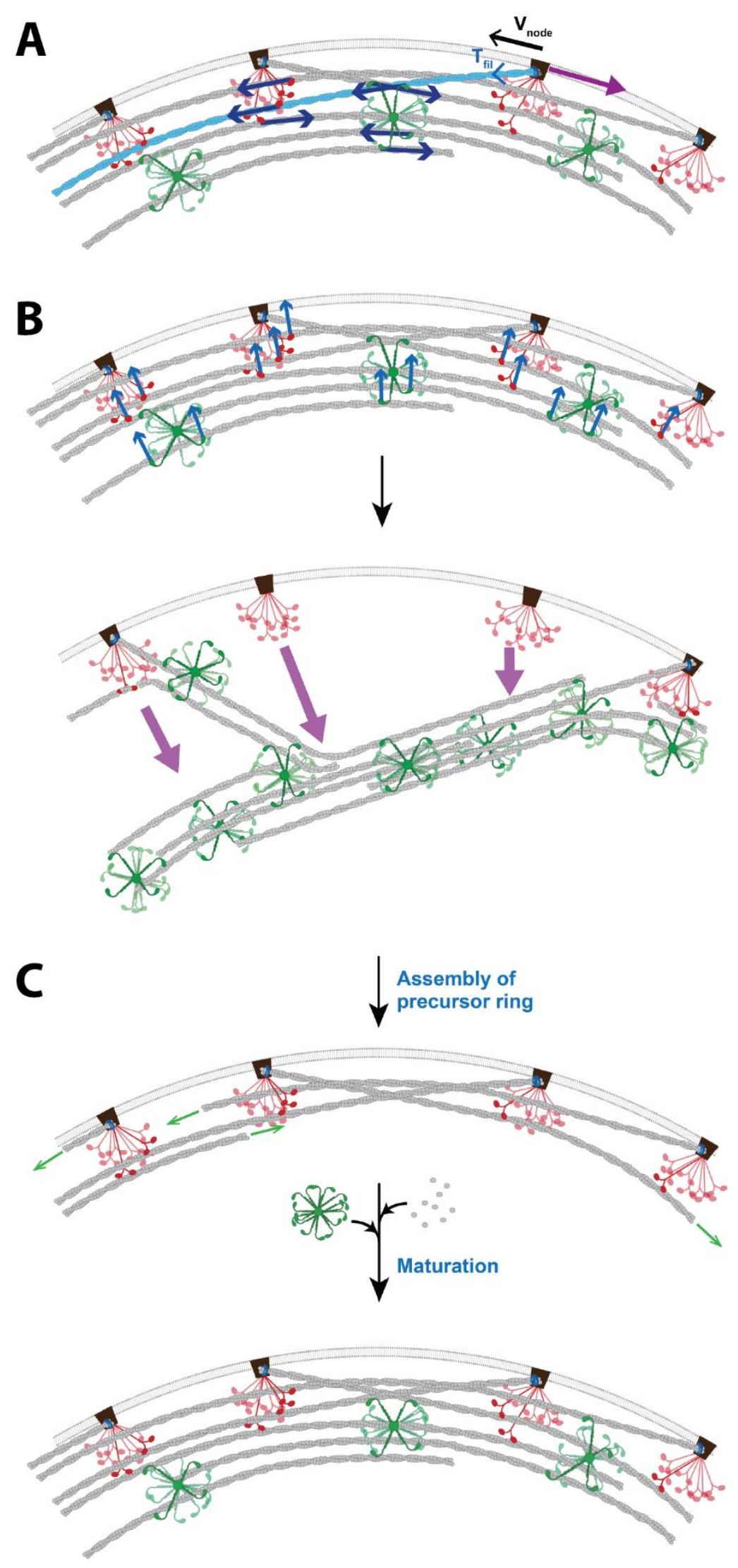
Figure 7. Model of the fission yeast contractile ring: assembly, anchoring and tension production

(A) Sliding node tension generation mechanism. Myo2 (red) and Myp2 (green) pull actin filaments whose barbed ends are anchored to the membrane by nodes. Large node-membrane drag forces (purple arrow) ensure nodes slide slowly $\left(v_{\text {node }}\right)$ and filaments develop tension $\left(T_{\text {fil }}\right)$. Unanchored Myp2 contributes due to the balanced bundle polarity.

(B) Myo2 and Myp2 cooperatively anchor and crosslink the actin filament bundle (forces, blue arrows) protecting the ring from bridging instabilities driven by the energetic preference of tense filaments to straighten. In myo2-E1 cells Myo2-actin binding is too weak to prevent bridging.

(C) Myo2 assembles the precursor ring from nodes. During maturation to the constriction-ready ring, actin filaments elongate and recruitment of unanchored Myp2 allows the ring to thicken. 